\title{
Elección de fármaco biológico en la psoriasis moderada-grave. Protocolo
}

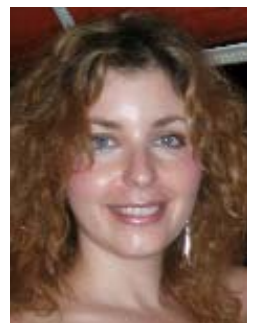

Elena González-Guerra Médico adjunto. Servicio de Dermatología. Hospital Clínico San Carlos. Madrid

\section{RESUMEN}

Hasta la fecha, se han publicado diferentes guías clínicas acerca del tratamiento de la psoriasis en placas moderada-grave. Sin embargo, no se ha publicado un protocolo que recoja los puntos que deben considerarse en la elección de fármacos biológicos en la psoriasis moderada-grave.

Las características basales de gravedad (PASI) de los pacientes no permiten por sí solas establecer a priori la selección del fármaco biológico. La presencia de características individuales del paciente o de la enfermedad y la forma de administración a menudo determinan el perfil de eficacia y seguridad de cada fármaco en cada paciente. Valoraremos, por tanto, en el proceso de selección de un determinado fármaco biológico, los factores que permiten tomar una decisión terapéutica individualizada, como son eficacia, seguridad, contraindicaciones, precauciones y otros datos.

Este protocolo debe ser revisado periódicamente, puesto que los datos recogidos a lo largo del tiempo sobre estas terapias aún jóvenes, el mayor número de pacientes en tratamiento, los datos de los registros (BIOBADADERM) todavía con escaso recorrido, los nuevos estudios y la incorporación de fármacos nuevos aportarán información que modificará la toma de decisión de tratamiento biológico en un futuro.

Palabras clave: protocolo, terapia biológica, guías clínicas, etanercept, adalimumab, infliximab, ustekinumab.

\section{ABSTRACT}

To date, various clinical guides have been published about the treatment of moderate-severe plaque psoriasis. However, no protocol has been published that summarizes the aspects to be considered when choosing the biologic drug for treatment of moderate-severe psoriasis.

The Psoriasis Area and Severity Index (PASI) does not by itself allow making an $a$ priori choice of biologic drug for patients. The individual characteristics of the patient or of the disease, as well as the form of administering the drug, often determine the efficacy and safety profile of each drug for each individual patient. Therefore, in the process of choosing a particular biologic drug, we will evaluate the factors that help decision making regarding individualized therapy, such as efficacy, safety, contraindications, precautions and other data.

This protocol should be reviewed regularly because the data collected over time about these still novel therapies, the larger number of patients receiving treatment, the data from records (BIOBADADERM) that have only recently been implemented, the new studies and the incorporation of new drugs will all provide more information that will modify future decision making about biologic treatment.

Keywords: protocol, biologic therapy, clinical guides, etanercept, adalimumab, infliximab, ustekinumab. 


\section{INTRODUCCIÓN}

Se define como guía clínica el conjunto de recomendaciones desarrolladas de manera sistemática, con objeto de guiar a los profesionales y a los pacientes en la toma de decisiones sobre qué intervenciones son más adecuadas en el abordaje de una condición clínica específica en circunstancias sanitarias concretas. Hasta la fecha, se han publicado diferentes guías clínicas acerca del tratamiento de la psoriasis en placas moderada-gra$\mathrm{ve}^{1-4}$. Sin embargo, no se ha publicado un protocolo sobre los puntos que deben considerarse en la elección de fármacos biológicos. Los protocolos representan la secuencia ordenada de actividades ante una situación clínica determinada que definen cómo ejecutarlas, con objeto de asegurar la presentación escalonada de procesos. Se trata de una serie de recomendaciones para la ejecución del acto médico expresadas de forma clara, concisa y ordenada, cuya misión es ayudar en la toma de decisiones clínicas y homogeneizar la calidad científico-técnica de la asistencia. El objetivo de este documento es la descripción del primer protocolo sobre el tratamiento con medicamentos biológicos en pacientes con psoriasis en placas moderada-grave.

\section{TRATAMIENTOS BIOLÓGICOS}

Las terapias biológicas están basadas en la administración exógena de diversos tipos de moléculas sintéticas relacionadas con la respuesta inmunitaria (anticuerpos $[\mathrm{AC}]$, receptores solubles, citocinas o antagonistas de las citocinas). Las terapias biológicas para la psoriasis se clasifican en dos grupos:

1. Agentes que se dirigen al factor de necrosis tumoral alfa (TNF $\alpha)$ : a) una proteína de fusión, etanercept; $b$ ) dos AC monoclonales, infliximab y adalimumab.

2. Agentes que interfieren específicamente en la diferenciación a Th1 y Th17 de las células T: ustekinumab.
PROPIEDADES DE LOS DISTINTOS FÁRMACOS BIOLÓGICOS

Véase tabla 1.

\section{INDICACIONES TERAPÉUTICAS ACTUALES DE LOS FÁRMACOS BIOLÓGICOS}

Las indicaciones clínicas formalmente aprobadas por la Agencia Europea de Medicamentos (EMA) para la administración de los tratamientos biológicos son el tratamiento de la psoriasis en placas de moderada a grave (PASI $\geq 10-12$ ) en los adultos que no responden, tienen contraindicadas o no toleran otras terapias sistémicas, incluyendo ciclosporina, metotrexato (MTX) y psoraleno más luz ultravioleta A (PUVA). Según la Agencia de Medicamentos y Alimentos (FDA), son tratamientos para pacientes adultos (mayores de 18 años) con psoriasis en placas de moderada a grave que sean candidatos a fototerapia o terapia sistémica, sin las restricciones en cuanto al tratamiento previo que incluye la EMA en su ficha técnica. En la tabla 2, se recogen las indicaciones terapéuticas y la fecha de aprobación de cada fármaco.

\section{DESARROLLO DE LA SELECCIÓN DEL FÁRMACO BIOLÓGICO}

Las características basales de gravedad (PASI) de los pacientes no permiten establecer a priori la selección del fármaco biológico. Las características individuales del paciente (peso, artritis, enfermedades concomitantes, contraindicaciones o situaciones que obligan a tener precauciones especiales, falta o pérdida de respuesta, previsión de tratamiento intermitente por viajes, embarazo, intervenciones, etc.) o de la enfermedad (rebote, extensión o carácter inflamatorio, que requieren una respuesta rápida) y la forma de administración a menudo determinan el perfil de eficacia y seguridad de cada fármaco en cada paciente. Valoraremos, por tanto, una larga serie de factores para tomar una decisión terapéutica individualizada (fig. 1). 
Tabla 1. Propiedades de los fármacos biológicos.

\begin{tabular}{|c|c|c|c|c|}
\hline & Etanercept & Adalimumab & Infliximab & Ustekinumab \\
\hline Estructura & $\begin{array}{l}\text { Receptor de TNF } \\
\text { soluble totalmente } \\
\text { humano }\end{array}$ & $\begin{array}{l}\text { AC monoclonal } \\
\text { humano }\end{array}$ & $\begin{array}{l}\text { AC monoclonal } \\
\text { quimérico }\end{array}$ & $\begin{array}{l}\text { AC monoclonal } \\
\text { totalmente humano }\end{array}$ \\
\hline $\begin{array}{l}\text { Presencia } \\
\text { de AC } \\
\text { neutralizantes }\end{array}$ & No & Sí & Sí & Sí \\
\hline Semivida & $<3$ días & 14 días & 8-9,5 días & 3 semanas \\
\hline $\begin{array}{l}\text { Fijación del } \\
\text { complemento }\end{array}$ & No & Sí & Sí & No \\
\hline $\begin{array}{l}\text { Diana } \\
\text { de unión }\end{array}$ & TNF y linfotoxina $\alpha$ & TNF & TNF & $\begin{array}{l}\text { Subunidad p40 de las } \\
\text { citocinas IL-12 e IL-23 }\end{array}$ \\
\hline Administración & $\begin{array}{l}25 \mathrm{mg} \text { sc } 2 \mathrm{v} / \mathrm{s} . \\
50 \mathrm{mg} 2 \mathrm{v} / \mathrm{s} \\
12 \mathrm{semanas} \text {, luego } \\
50 \mathrm{mg} 1 \mathrm{v} / \mathrm{s} \text {. } \\
\text { En terapia intermitente, } \\
\text { se siguen las mismas } \\
\text { pautas de tratamiento. } \\
\text { A partir de } 8 \text { años de } \\
\text { edad, es de } 0,8 \mathrm{mg} / \mathrm{kg} \\
\text { hasta un máximo } \\
\text { de } 50 \mathrm{mg} \text { por dosis } \\
1 \mathrm{v} / \mathrm{s} .\end{array}$ & $\begin{array}{l}80 \text { mg en la semana } \\
\text { 0, sc seguida de } 40 \mathrm{mg} \\
\text { administrados } \\
\text { en semanas alternas, } \\
\text { empezando una } \\
\text { semana después } \\
\text { de la dosis inicial. }\end{array}$ & $\begin{array}{l}5 \mathrm{mg} / \mathrm{kg} \text { en infusión } \\
\text { intravenosa seguida } \\
\text { de infusión a las } 2 \text { y } \\
6 \text { semanas siguientes } \\
\text { a la primera } \\
\text { y posteriormente cada } \\
8 \text { semanas. }\end{array}$ & $\begin{array}{l}\text { En pacientes con peso } \\
<100 \mathrm{~kg}: 45 \mathrm{mg} \text { en } \\
\text { la semana } 0, \text { semana } \\
4 \text { y después cada } \\
12 \text { semanas. } \\
\text { En pacientes con peso } \\
>100 \mathrm{~kg}: 90 \mathrm{mg} \text { en } \\
\text { la semana } 0, \text { semana } \\
4 \text { y después cada } \\
12 \text { semanas. }\end{array}$ \\
\hline
\end{tabular}

AC: anticuerpo; TNF: factor de necrosis tumoral; v/s: veces por semana.

Tabla 2. Indicaciones terapéuticas y fecha de aprobación de los fármacos biológicos.

\begin{tabular}{|c|c|c|c|c|}
\hline \multirow{2}{*}{$\begin{array}{l}\text { Fármaco } \\
\text { biológico }\end{array}$} & \multicolumn{3}{|c|}{ Fecha de aprobación para psosiasis moderada-grave } & \multirow{2}{*}{$\begin{array}{l}\text { Otras indicaciones } \\
\text { terapéuticas }\end{array}$} \\
\hline & $\begin{array}{l}\text { En terapia } \\
\text { continua }\end{array}$ & \begin{tabular}{|l|} 
En terapia \\
intermitente
\end{tabular} & $\begin{array}{l}\text { En niños } \\
\text { ( }>8 \text { años })\end{array}$ & \\
\hline Etanercept & Septiembre de 2009 & Diciembre de 2004 & Febrero de 2009 & AR, EA, AP, AIJ. \\
\hline Adalimumab & Diciembre de 2007 & & & AR, AIJ poliarticular, EA, AP, EC. \\
\hline Infliximab & Septiembre de 2005 & & & $\begin{array}{l}\text { AR, EC, EC pediátrica, colitis } \\
\text { ulcerosa, EA, AP. }\end{array}$ \\
\hline Ustekinumab & Febrero de 2009 & & & $\begin{array}{l}\text { Estudios en fase II en EII. } \\
\text { Estudios en fase III en AP. }\end{array}$ \\
\hline
\end{tabular}

AIJ: artritis idiopática juvenil; AP: artritis psoriásica; AR: artritis reumatoide; EA: espondiloartritis anquilosante; EC: enfermedad de Crohn; EII: enfermedad inflamatoria intestinal.

\section{Eficacia de los tratamientos biológicos}

Datos de eficacia en niños. La reciente autorización del etanercept con indicación en el tratamiento de la psoriasis grave en placas en la población pediátrica se basa en los resultados obtenidos en el primer ensayo clínico publicado en 2008. En la semana 12 , el $57 \%$ de los pacientes que recibieron etanercept $(0,8 \mathrm{mg} / \mathrm{kg} / \mathrm{semana})$ alcanzaron un PASI 75, en comparación con el $11 \%$ que recibieron placebo $(p<0,001)$. Durante el período abierto, alcanzaron un PASI 75 en la semana 24 el $62 \%$ de los pacientes del grupo de placebo original y el $69 \%$ de los pacientes del grupo de eta- 
Paciente con psoriasis en placas de moderada a grave (PASI $\geq 10-12$ ) en los adultos que no responden, tienen contraindicadas o no toleran otras terapias sistémicas, incluyendo ciclosporina, metotrexato y PUVA.

\begin{tabular}{|c|c|c|c|}
\hline & 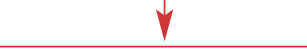 & 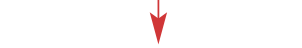 & $\downarrow$ \\
\hline Edad pediátrica (> 8 años) & Con artritis psoriásica & Terapia intermitente & Terapia continua \\
\hline & 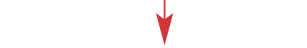 & $y$ & $\downarrow$ \\
\hline \multirow[t]{4}{*}{ Etanercept } & $\begin{array}{l}\text { 1a línea de tratamiento } \\
\text { Etanercept } \\
\text { Adalimumab } \\
\text { Infliximab }\end{array}$ & $\begin{array}{l}\text { 1a línea de tratamiento } \\
\text { Etanercept }\end{array}$ & $\begin{array}{l}\text { 1a línea de tratamiento } \\
\text { Etanercept } \\
\text { Adalimumab } \\
\text { Infliximab } \\
\text { Ustekinumab }\end{array}$ \\
\hline & $\downarrow$ & 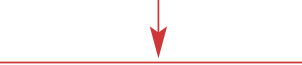 & $\downarrow$ \\
\hline & $\begin{array}{l}2^{\text {a }} \text { línea de tratamiento } \\
\text { Ustekinumab }\end{array}$ & $\begin{array}{l}2^{\text {a }} \text { línea de tratamiento } \\
\text { Adalimumab } \\
\text { Ustekinumab }\end{array}$ & $\begin{array}{l}2^{\text {a }} \text { línea de tratamiento } \\
\text { Etanercept } \\
\text { Adalimumab } \\
\text { Infliximab } \\
\text { Ustekinumab }\end{array}$ \\
\hline & & $\Lambda$ & $\uparrow$ \\
\hline \multicolumn{4}{|c|}{$\begin{array}{l}\text { Otros factores que influyen en la elección del fármaco: } \\
\text { - Mecanismo de acción del fármaco. } \\
\text { - Fecha de aprobación del fármaco. } \\
\text { - Datos de eficacia. } \\
\text { - Datos de seguridad. } \\
\text { - Contraindicaciones absolutas de cada fármaco. } \\
\text { - Precauciones y situaciones especiales: } a \text { ) insuficiencia cardíaca, b) infecciones (VHC, VHB, HIV, etc.), c) embarazo } \\
\text { y lactancia, } d \text { ) tumores malignos (neoplasias hematológicas, tumores sólidos, tumores cutáneos), e) vacunas, ancia- } \\
\text { nos, cirugía } d \text { ) interacciones con otros fármacos, e) enfermedades desmielinizantes, f) alteraciones hematológicas, } \\
\text { g) sensibilidad al látex, } h \text { ) procesos autoinmunes, i) otros datos (médico prescriptor, obesidad, coste). }\end{array}$} \\
\hline
\end{tabular}

Figura 1. Árbol de decisión de terapia biológica en el caso de un paciente con psoriasis candidato a terapia biológica en edad pediátrica, que asocie además artritis psoriásica o cuando lo más indicado sea la terapia intermitente o continua.

nercept original ${ }^{5}$. Las respuestas PASI 50, 75 y 90 fueron del $89 \%, 61 \%$ y $30 \%$, respectivamente, durante el período de extensión de 96 semanas ${ }^{6}$.

Datos de eficacia en adultos en tratamiento en terapia intermitente (con pausas). Con etanercept (único fármaco aprobado en terapia intermitente), el 93\% de los pacientes que alcanzaron una respuesta PASI 75 en la semana 12 del primer ciclo de tratamiento lograron una respuesta PASI 50 al finalizar la semana 12 del ciclo de retratamien- to $^{7,8}$. El etanercept ha demostrado una eficacia similar tanto en terapia continua como intermitente; sin embargo, la administración continua se asocia con un control más estable de la enferme$\operatorname{dad}^{9,10,11}$.

Datos de eficacia en adultos en tratamiento con terapia continua (tabla 3). Solo existe un metanálisis de todos los fármacos biológicos disponibles en la actualidad, en cuanto a eficacia en la semana $12^{12}$. Los datos obtenidos en la semana 24 
Tabla 3. Datos de eficacia de los tratamientos biológicos en psoriasis moderada-grave en terapia continua.

\begin{tabular}{|c|c|c|c|c|c|c|}
\hline & $\begin{array}{l}\text { Etanercept } \\
25 \mathrm{mg} 2 \mathrm{v} / \mathrm{s} \\
050 \mathrm{mg} 1 \mathrm{v} / \mathrm{s}\end{array}$ & $\begin{array}{l}\text { Etanercept } \\
50 \mathrm{mg} 2 \mathrm{v} / \mathrm{s} \\
12 \text { semanas, } \\
\text { seguido de } \\
50 \mathrm{mg} 1 \mathrm{v} / \mathrm{s}\end{array}$ & Adalimumab & $\begin{array}{l}\text { Ustekinumab } \\
90 \mathrm{mg}\end{array}$ & $\begin{array}{l}\text { Ustekinumab } \\
45 \text { mg }\end{array}$ & Infliximab \\
\hline $\begin{array}{l}\text { Eficacia semana } \\
12 \text { PASI } 75\end{array}$ & $\begin{array}{l}34 \% * \\
(2)\end{array}$ & $49 \% *-55 \%$ & $68 \%$ & $69 \%$ & $74 \%$ & $80 \%$ \\
\hline $\begin{array}{l}\text { Eficacia PASI } \\
75 \text { a las } \\
24 \text { semanas } \\
\end{array}$ & $44-71 \%(1)$ & $78 \%(3)$ & $70,3 \%$ & $74 \%$ & $83 \%$ & $82,2 \%$ \\
\hline $\begin{array}{l}\text { Eficacia a largo } \\
\text { plazo } \\
\text { PASI } 75\end{array}$ & Sin datos & $\begin{array}{l}63 \% \\
\text { semana } 48 \\
47 \%-60 \% \\
2,5 \text { años }\end{array}$ & $\begin{array}{l}76 \% \\
\text { semana } 160\end{array}$ & $\begin{array}{l}72 \% \\
\text { semana } \\
40-148\end{array}$ & $\begin{array}{l}88 \% \\
\text { semana } \\
40-148\end{array}$ & $\begin{array}{l}61 \% \\
\text { semana } 50\end{array}$ \\
\hline $\begin{array}{l}\text { Inicio de efecto } \\
\text { clínico habi- } \\
\text { tualmente en: }\end{array}$ & \multicolumn{2}{|l|}{ 4-8 semanas } & \multicolumn{2}{|l|}{ 2-4 semanas } & 2-4 semanas & $\begin{array}{l}1-2 \\
\text { semanas }\end{array}$ \\
\hline $\begin{array}{l}\text { Duración } \\
\text { de la remisión }\end{array}$ & 70-80 días & Sin datos & 141 días & 18,1 semanas & 14,4 semanas & 140 días \\
\hline $\begin{array}{l}\text { Eficacia } \\
\text { en artritis } \\
\text { psoriásica }\end{array}$ & \multicolumn{2}{|c|}{$\begin{array}{l}\text { Buena eficacia. El tratamiento } \\
\text { a largo plazo reduce } \\
\text { la progresión de la destrucción } \\
\text { ósea. }\end{array}$} & \multicolumn{2}{|c|}{$\begin{array}{l}\text { Buena eficacia. Reduce la } \\
\text { progresión de la destrucción } \\
\text { ósea. }\end{array}$} & \multicolumn{2}{|c|}{ Ensayo clínico en fase III. } \\
\hline
\end{tabular}

v/s: veces por semana.

son un comparativo en el que se extraen datos obtenidos de diversos ensayos clínicos ${ }^{13-22}$. La eficacia a largo plazo es muy difícil de comparar entre los distintos tratamientos, dados los diferentes diseños de los estudios y el bajo número de pacientes que en muchos casos llegan al final para análisis PASI 75.

Efecto de la retirada del fármaco. Ninguno de los cuatro fármacos biológicos aprobados para el tratamiento de la psoriasis en placas moderadagrave ha demostrado tener un efecto rebote. Los datos sobre la duración de la remisión (tiempo transcurrido hasta la reaparición de la enfermedad) están recogidos en la tabla 3.

\section{Efecto del retratamiento con:}

Adalimumab: con la dosis inicial de $80 \mathrm{mg}$, ha demostrado que el $69 \%$ de los pacientes respondedores recuperan la respuesta previa ${ }^{21}$. En el estu- dio REVEAL, se observó que la recuperación de la respuesta durante el retratamiento fue mejor en aquellos pacientes que conservaban un PASI $\geq 50$ durante la interrupción del tratamiento ${ }^{22}$.

Infliximab: la respuesta a tandas de inducción repetida o a tratamiento intermitente no es tan buena como la que se consigue con la primera tanda de tres inyecciones y puede asociarse a una mayor incidencia de reacciones de infusión, mayor riesgo de desarrollar una resistencia al tratamiento o pérdida de eficacia del mismo (y AC antiinfliximab) ${ }^{23}$.

Ustekinumab: en el ensayo PHOENIX 1, se retrató a los pacientes que, tras suspensión de ustekinumab, habían perdido al menos el $50 \%$ de la mejoría obtenida en el PASI. Tras reiniciar el tratamiento con ustekinumab, el 85,6\% de los pacientes alcanzaron una respuesta PASI 75 en un plazo de 12 semanas ${ }^{15,24}$. 


\section{Datos de seguridad}

\section{de los tratamientos biológicos}

Según los resultados de BIOBADADERM, los tratamientos biológicos presentan un mayor número de acontecimientos adversos y más graves que los controles. Destacan las infecciones e infestaciones, los trastornos de la piel y el tejido subcutáneo y los trastornos de la sangre y del sistema linfático ${ }^{25}$.

Etanercept. Durante el primer ensayo clínico en niños y adolescentes con psoriasis en placas moderada-grave, no se notificaron casos de muerte, cáncer, infecciones oportunistas, tuberculosis (TB) ni enfermedades desmielinizantes ${ }^{5,6}$. En adultos, ha demostrado el mismo perfil de seguridad favorable en tratamientos de hasta 4,2 años $7,9-11,17,26,27$.

Adalimumab. Un análisis integrado de seguridad a largo plazo de los ensayos clínicos en psoriasis indica que la incidencia de acontecimientos adversos, de infecciones e infecciones graves y de acontecimientos adversos de interés fueron por lo general bajos y estables a lo largo del tiempo ${ }^{28}$.

Infliximab. Los efectos adversos más frecuentes del tratamiento con infliximab son cefaleas, náuseas, infecciones repiratorias de vías altas y reacciones de infusión (en un 3,8 a un $27 \%$ de los pacientes), aunque la mayoría de las reacciones de infusión son leves y está bien protocolizado su tratamiento. En el ensayo clínico EXPRESS, la tasa de reacciones de infusión graves (que precisaron la retirada del tratamiento) fue de $1 \%^{29}$.

Ustekinumab. Los acontecimientos adversos notificados durante un máximo de 4 años fueron en su mayoría leves ${ }^{30}$ y se mantuvieron estables a lo largo del tiempo, sin evidencias de toxicidad acumulada, ni riesgos asociados a una exposición mayor ${ }^{30}$.

\section{Contraindicaciones absolutas}

Las contraindicaciones que figuran en la ficha técnica comunes a etanercept, ustekinumab, adalimumab e inflixmab son:

\section{a. Hipersensibilidad al principio activo o a alguno de sus excipientes.}

\section{b. Infecciones activas, incluyendo infecciones cró- nicas o localizadas, hasta que no estén contro- ladas.}

Las contraindicaciones específicas de infliximab y adalimumab son, además:

a. Insuficiencia cardíaca moderada-grave (según la clasificación de NYHA clases III/IV).

b. Pacientes con tuberculosis. El incremento de la susceptibilidad a desarrollar TB o de reactivación de una TB latente debe considerarse como una característica específicamente asociada al uso de anti-TNF. En el caso de la TB, suele tratarse de la reactivación de una infección latente $^{31}$. Es frecuente la presentación extrapulmonar y la forma diseminada entre el $2 .^{\circ}$ y el $5 .^{\circ}$ mes de tratamiento con anti-TNF. Se han descrito más casos de TB en pacientes tratados con infliximab y adalimumab que con etanercept ${ }^{31}$ y ustekinumab (con el que no se ha descrito ningún caso hasta la fecha).

\section{Precauciones y situaciones especiales}

a. Insuficiencia cardíaca. Se ha demostrado una asociación de la psoriasis con factores de riesgo predictivos de enfermedad cardiovascular (CV) aterosclerótica ${ }^{32}$. Amplios estudios prospectivos han demostrado que la psoriasis en sí misma podría suponer un riesgo independiente de infarto de miocardio (IM), ictus, muerte por causa CV y mortalidad general ${ }^{33,34}$. Sin embargo, los efectos del ustekinumab en los factores de riesgo CV en los ensayos clinicoanalíticos no son significativos en cuanto a tensión arterial, frecuencia cardíaca, peso, electrocardiograma, lípidos y perfil de glucosa. A largo plazo, la tasa de IM y accidente CV es menor que la tasa esperada en la población general $($ SIR 0,52$)$ y menor que la esperada en la población psoriásica $(0,34)^{35}$.

b. Infecciones. Todo tratamiento que modifique el sistema inmunitario podría incrementar el 
riesgo de infección. Se deben extremar las precauciones cuando se considere el uso de tratamientos biológicos en pacientes con historial de infecciones crónicas o recurrentes, o con trastornos subyacentes que pudieran predisponer a las infecciones, tales como diabetes avanzada o mal controlada. Las infecciones graves descritas entre pacientes tratados con anti-TNF son: TB pulmonar y extrapulmonar, herpes zóster, infecciones respiratorias profundas (legionelosis, infecciones por listeria, artritis séptica). En el caso del ustekinumab, en los ensayos clínicos, se han observado infecciones bacterianas, fúngicas y víricas graves. Las personas con déficit genético en la vía IL12/23 tienen una mayor incidencia de infecciones por micobacterias y salmonela; sin embargo, no se han descrito casos en los pacientes tratados con ustekinumab ${ }^{36,37}$.

- Hepatitis B. Se ha notificado la reactivación del virus de la hepatitis B (VHB) en pacientes crónicos portadores de este virus que están en tratamiento con antagonistas del TNF (etanercept, adalimumab, infliximab) ${ }^{38}$. Si se utilizan estos fármacos en pacientes portadores de VHB, se deben monitorizar para detectar síntomas y signos de una infección activa durante todo el tratamiento y hasta varios meses después y, si es necesario, se debe iniciar un tratamiento apropiado. No existen datos de reactivación de la hepatitis $\mathrm{B}$ en el caso del ustekinumab.

- Hepatitis C. El uso de agentes anti-TNF no parece estar asociado a variaciones en la carga viral, elevación de las transaminasas, ni a un aumento de efectos adversos ${ }^{38-41}$. Tampoco se han detectado cambios en la hepatopatía crónica en los dos casos descritos de pacientes $\mathrm{VHC}+$ en tratamiento con ustekinumab ${ }^{42}$.

- Infección por VIH. La infección por VIH es una de las situaciones en las que la terapia biológica debe utilizarse con precaución, y las decisiones deben tomarse de forma individualizada.

c. Embarazo y lactancia. No se dispone de datos clínicos suficientes sobre embarazos y adminis- tración de fármacos biológicos. Los anti-TNF están clasificados como categoría B (toxicidad en humanos no documentada) por la FDA. En las primeras 12 semanas de gestación, cabe considerar que el paso transplacentario de una molécula de IgG es despreciable, por lo que la recomendación en el caso de AC monoclonales debería ser suspender el tratamiento a partir del conocimiento de la situación de embarazo, para evitar el paso transplacentario del AC monoclonal a partir del segundo trimestre de gestación ${ }^{43}$. Tampoco hay datos sobre la seguridad del embarazo en mujeres cuya pareja esté en tratamiento con un fármaco biológico.

No se conoce el grado en el que los anti-TNF se excretan por la leche humana. Un único estudio muestra que solo el $0,0001 \%$ de la concentración plasmática del etanercept se encuentra en la leche materna ${ }^{44}$. Un caso clínico demostró que el adalimumab se excreta por la leche materna en concentraciones bajas. A pesar de ello, no se recomienda la administración de terapia biológica a mujeres en período de lac$\operatorname{tancia}^{45}$.

\section{d. Tumores malignos:}

- Neoplasias sólidas. Aunque diversos estudios no han mostrado una mayor incidencia de neoplasias sólidas en pacientes con psoriasis tratados con terapia anti-TNF, no hay suficiente información sobre el desarrollo de nuevas neoplasias en pacientes con antecedente de neoplasia curada ${ }^{46}$. Las tasas y el patrón de neoplasias malignas observadas en los pacientes tratados con ustekinumab durante 3 años fueron coherentes con las tasas esperadas en la población general de Estados Unidos ${ }^{47}$. Se debe tener especial cuidado en pacientes con un riesgo elevado de sufrir enfermedades malignas, como, por ejemplo, con hábito tabáquico.

- Neoplasias hematológicas. Se han observado más casos de linfomas entre los pacientes que recibieron un antagonista del TNF (infliximab, adalimumab y etanercept) en comparación 
con el grupo de control, en las partes controladas de los ensayos clínicos ${ }^{25}$. Aun así, la incidencia fue baja ${ }^{25}$. Con la administración de ustekinumab, no se ha observado aumento en las tasas de linfoma respecto a los pacientes tratados con placebo ${ }^{47}$.

- Tumores cutáneos: en un reciente estudio donde se analiza la seguridad del tratamiento con fármacos biológicos anti-TNF en la población con psoriasis, en la práctica diaria a lo largo de 5 años, se ha observado un aumento significativo de la incidencia de cáncer de piel no melanoma (NMSC) en la población tratada frente a la población general ${ }^{46}$. En el caso del ustekinumab, no se han observado tasas más altas de NMSC y melanoma con ustekinumab que con placebo ${ }^{47}$.

- Vacunas: no existe información disponible en relación con la respuesta a la vacunación con virus vivo ni con la transmisión secundaria de la infección causada por virus vivo en pacientes que están recibiendo terapia con fármacos biológicos. Por tanto, debe evitarse.

- Ancianos: no se apreciaron diferencias globales en la seguridad o eficacia entre los pacientes de mayor edad tratados con fármacos biológicos incluidos en los ensayos clínicos y los más jóvenes. Debido a la mayor incidencia de infecciones y de enfermedades cardiopulmonares en las personas de la tercera edad en general, se actuará con cautela al tratar con fármacos biológicos a este grupo de edad.

- Cirugía: se recomienda suspender el tratamiento con anti-TNF entre 2 y 4 semanas antes de la intervención ${ }^{48}$. En el caso del ustekinumab, se recomienda esperar cinco semividas antes de la intervención quirúrgica, según datos de la ficha técnica.

- Interacciones con otros fármacos: la combinación de abatacept o anakinra con etanercept no ha demostrado que incremente el beneficio clínico, pero sí produce un aumento de reacciones adversas graves. Por la naturaleza de los efectos adversos observados, la combina- ción de anakinra o abatacept y otros antagonistas del TNF puede producir una toxicidad similar. Por tanto, no se recomienda la combinación de antagonistas del TNF con anakinra o abatacept, según datos de la ficha técnica. En el caso del ustekinumab, se ha investigado el efecto de los medicamentos concomitantes más utilizados en los pacientes con psoriasis (incluidos paracetamol, ibuprofeno, ácido acetilsalicílico, metformina, atorvastatina y levotiroxina), sin que se encontrasen indicios de interacciones ${ }^{15}$.

- Enfermedades desmielinizantes: se han descrito casos aislados de aparición o exacerbación de enfermedades desmielinizantes del sistema nervioso central (como la esclerosis múltiple, la neuritis óptica y la mielitis transversa) en relación con el uso de agentes anti-TNF. Todos los casos experimentaron mejoría al interrumpir el tratamiento ${ }^{42}$. Durante los ensayos con ustekinumab, no se registró ningún caso de enfermedad desmielinizante. Incluso se inició un ensayo a doble ciego para el tratamiento de la esclerosis múltiple con ustekinumab ${ }^{49}$.

- Alteraciones hematológicas: en raras ocasiones, se han descrito casos de pancitopenias, así como de citopenias significativas desde el punto de vista médico (por ejemplo, trombocitopenia) en los pacientes tratados con anti-TNF. El mecanismo podría estar relacionado con un efecto colateral de inhibición de la función de las interleucinas 1, 6 y 8 , así como de factores estimulantes de colonias ${ }^{50}$. En el caso del ustekinumab, no consta en su ficha técnica ninguna advertencia especial del uso en pacientes con leucopenia.

- Sensibilidad al látex: el capuchón de la aguja de la jeringa del diluyente de etanercept, de la pluma precargada de adalimumab y de la jeringa precargada de ustekinumab contiene látex. Esto puede causar reacciones de hipersensibilidad en personas con sensibilidad conocida o posibles antecedentes de sensibi- 
lidad al látex. En el caso del infliximab, se administra mediante infusión y no existe contacto con látex.

- Procesos autoinmunes: todos los tratamientos biológicos son proteínas recombinantes ajenas al organismo y, por tanto, pueden inducir la aparición de AC y otros fenómenos inmunitarios. Las reacciones inmunológicas pueden agruparse en:

a) Producción de AC anti-TNF: los AC antietanercept aparecen con una incidencia aproximada del $6 \%$, aunque no son neutralizantes y su presencia no se relaciona con la aparición de efectos adversos o pérdida de eficacia ${ }^{42}$. Se han identificado AC antiadalimumab en el 8,4\% de los pacientes con psoriasis tratados con adalimumab en monoterapia, sin que se relacionen con la presencia de efectos adversos. La formación de AC antiadalimumab se asocia con una disminución de la eficacia ${ }^{22}$. En los pacientes con psoriasis en placas en monoterapia con adalimumab a largo plazo que participaron en el estudio de retirada y retratamiento, el porcentaje de AC para adalimumab después del retratamiento (11 de 482 sujetos, $2,3 \%)$ fue similar al observado antes de la retirada (11 de 590 sujetos, $1,9 \%{ }^{22}$. Con elevada frecuencia, se desarrollan AC antiinfliximab, que pueden reducir la duración del efecto terapéutico y aumentar las reacciones a la infusión; esto es más frecuente en tratamientos intermitentes que con pauta constante ${ }^{51}$. La asociación con MTX o con otros inmunosupresores (azatioprina, corticoides, etc.) disminuye la producción de AC. Alrededor del $5 \%$ de los pacientes tratados con ustekinumab desarrollaron AC antiustekinumab, generalmente a títulos bajos, sin que se observe relación aparente con el aumento de reacciones en el lugar de inyección. La eficacia tiende a ser menor en pacientes con AC positivos frente a ustekinumab ${ }^{52}$.
En los pacientes de más de $100 \mathrm{~kg}$, se observa un mayor porcentaje de AC antiustekinumab cuando se utilizan dosis de $45 \mathrm{mg}^{53}$.

b) Auto-AC: el $11 \%$ de los pacientes tratados con etanercept desarrollaron positividad para AC antinucleares (ANA) y el $15 \%$ para anti$\mathrm{ADN}$ de doble filamento (anti- $\mathrm{ADNds}{ }^{42}$. El tratamiento con adalimumab puede dar lugar a la formación de auto-AC ${ }^{54}$. En los pacientes tratados con infliximab, se han observado ANA en un 52\% de los $\operatorname{casos}^{51}$, AC anti-ADNds (17\%) y anticardiolipina ${ }^{51,55}$. Se desconoce el impacto del tratamiento a largo plazo sobre el desarrollo de enfermedades autoinmunes. Si un paciente desarrolla síntomas parecidos al lupus y da positivo a los AC frente al ADNds, se debe interrumpir el tratamiento. No existen datos sobre el ustekinumab y la producción de auto-AC.

c) Reacciones inmunológicas retardadas: son reacciones que ocurren en un $2 \%$ de los casos, 5 a 9 días después de la infusión de infliximab en pacientes en los que se ha inducido un período de descanso de 12-50 semanas (tratamientos intermitentes) ${ }^{55}$.

\section{Otros datos que intervienen en la decisión de administrar fármacos biológicos}

1. El médico prescriptor. Tanto el manejo de los distintos tratamientos biológicos por parte del médico como las experiencias previas obtenidas con estos fármacos influyen en la elección de uno u otro tratamiento.

2. La obesidad. Solo son dos los tratamientos biológicos con dosis ajustadas al peso, infliximab y ustekinumab. En un estudio, se observa que una respuesta óptima es menos frecuente en los pacientes obesos, fundamentalmente por encima de los $100 \mathrm{~kg}$, cuando se utilizan los fármacos biológicos a dosis fijas independientemente del peso ${ }^{56}$.

3. El coste. Las consideraciones económicas constituyen un importante elemento de juicio, pero 
deben ser ajenas a la decisión terapéutica. Para valorar la eficiencia real de las diferentes opciones de tratamiento según la práctica clínica habitual, hay que tener en consideración otros factores importantes, además del coste de adquisición (coste de inducción, de mantenimiento, paciente respondedor, etc.).

Este protocolo debe ser revisado periódicamente, puesto que los datos recogidos a lo largo del tiempo sobre estas terapias aún jóvenes, el mayor número de pacientes en tratamiento, los datos de los registros (BIOBADADERM) todavía con escaso recorrido, nuevos estudios y la incorporación de fármacos nuevos aportan información que modificará la toma de decisión de tratamiento biológico en un futuro.

\section{BIBLIOGRAFÍA}

1. Papp K, Gulliver W, Lynde C, Poulin Y, Ashkenas J. Canadian guidelines for the management of plaque psoriasis: overview. J Cutan Med Surg. 2011;15(4):210-9.

2. Foulkes AC, Grindlay DJ, Griffiths CE, Warren RB. What's new in psoriasis? An analysis of guidelines and systematic reviews published in 2009-2010. Clin Exp Dermatol. 2011;36(6): 585-9.

3. Menter A, Korman NJ, Elmets CA, Feldman SR, Gelfand JM, Gordon KB, et al. Guidelines of care for the treatment of psoriasis and psoriatic arthritis: Case-based presentations and evidence-based conclusions. J Am Acad Dermatol. 2011;65(1): 137-74.

4. Ormerod AD, Campalani E, Goodfield MJ, BAD Clinical Standards Unit. British Association of Dermatologists guidelines on the efficacy and use of acitretin in dermatology. $\mathrm{Br}$ J Dermatol. 2010;162(5):952-63.

5. Paller AS, Siegfried EC, Langley RG, Gottlieb AB, Pariser D, Landells I, et al. Etanercept treatment for children and adolescents with plaque psoriasis. N Engl J Med. 2008;358(3):241-51.

6. Paller AS, Siegfried EC, Eichenfield LF, Pariser D, Landells I, et al. Long-term etanercept in pediatric patients with plaque psoriasis. J Am Acad Dermatol. 2011;64(1):64-70.

7. Gordon KB, Gottlieb AB, Leonardi CL, Elewski BE, Wang A, Jahreis $A$, et al. Clinical response in psoriasis patients discontinued from and then reinitiated on etanercept therapy. J Dermatol Treat. 2006;17(1):9-17.

8. Ortonne JP, Taïeb A, Ormerod AD, Robertson D, Foehl J, Pedersen $\mathrm{R}$, et al. Patients with moderate-to-severe psoriasis recapture clinical response during re-treatment with etanercept. Br J Dermatol. 2009;161(5):1190-5.

9. Ortonne JP, Griffiths C, Daudén E, Strohal R, Robertson D, Pedersen $\mathrm{R}$, et al. Efficacy and safety of continuous versus paused etanercept treatment in patients with moderate-to-severe psoriasis over 54 weeks: the CRYSTEL study. Expert Rev Dermatol. 2008;3(6):657-65.

10. Camacho F, Puig L, Gimeno E, Gállego M, López-Ávila A, García-Calvo C. Efectividad de etanercept en psoriasis moderada-severa. Un año de seguimiento. [Poster] en AEDV 2009.
11. Puig L, Camacho F, Gimeno E, Gállego M, López-Ávila A, García-Calvo C. Seguridad de etanercept en psoriasis moderada-severa un año de seguimiento. [Póster] en AEDV 2009.

12. Hawkins N, Eaton J. Weight-based meta-analysis of biologic therapies for the treatment of moderate to severe psoriasis. [Póster] en 9o Simposio de CPES en Sao Paul 2010.

13. Puig L. Eficacia comparativa de ustekinumab, etanercept y adalimumab en el manejo de la psoriasis moderada-grave. [Póster] en 38 congreso AEDV, 2010, Málaga.

14. Griffiths C, Strober BE, van de Kerkhof P, Ho V, Fidelus-Gort $\mathrm{R}$, Yeilding $\mathrm{N}$, et al. Comparison of ustekinumab and etanercept for moderate-to-severe psoriasis. N Engl J Med. 2010; 362(2):118-28.

15. Leonardi CL, Kimball AB, Papp KA, Yeilding N, Guzzo C, Wang $Y$, et al. Efficacy and safety of ustekinumab, a human interleukin-12/23 monoclonal antibody, in patients with psoriasis: 76-week results from a randomised, double-blind, placebocontrolled trial (PHOENIX 1). Lancet. 2008;371:1665-74.

16. Menter A, Tyring SK, Gordon K, Kimball AB, Leonardi CL, Langley RG, et al. Adalimumab therapy for moderate to severe psoriasis: a randomized, controlled phase III trial. J Am Acad Dermatol. 2008;58(1):106-15.

17. Papp KA, Tyring S, Lahfa M, Prinz J, Griffiths CE, Nakanishi AM, et al. A global phase III randomized controlled trial of etanercept in psoriasis: safety, efficacy, and effect of dose reduction. Br J Dermatol. 2005;152(6):1304-12.

18. Van de Kerkhof PC, Segaert S, Lahfa M, Luger TA, Karolyi Z, Kaszuba A, et al. Once weekly administration of etanercept 50 $\mathrm{mg}$ is efficacious and well tolerated in patients with moderateto-severe plaque psoriasis: a randomized controlled trial with open-label extension. Br J Dermatol. 2008;159(5):1177-85.

19. Sterry W, Ortonne JP, Kirkham B, Brocq O, Robertson D, Pedersen RD, et al. Comparison of two etanercept regimens for treatment of psoriasis and psoriatic arthritis: PRESTA randomised double blind multicentre trial. BMJ. 2010;340:c147.

20. Strohal R, Puig L, Roberston D, Estojak J, Ciesielska M, Pedersen $R$, et al. Efficacy and safety of etanercept for the treatment of moderate-to-severe psoriasis when used with adjunctive therapy as needed: the PRISTINE Trial. Abstract 404 in 40th Annual ESDR Meeting; 8-11 septiembre 2010, Helsinki, Finlandia.

21. Papp K, Growley J, Ortonne JP, Leu J, Okun M, Gupta SR, et al. Adalimumab for moderate to severe chronic plaque psoriasis: efficacy and safety of retreatment an disease recurrence following withdrawal from therapy. Br J Dermatol. 2011; 164(2):434-41.

22. Gordon K, Papp K, Poulin Y, Gu Y, Rozzo S, Sasso EH. Longterm efficacy and safety of adalimumab in patients with moderate to severe psoriasis treated continuously over 3 years: Results from an open-label extension study for patients from REVEAL. J Am Acad Dermatol. 2012;66(2):241-51.

23. Rich $P$, Griffiths CE, Reich K, Nestle FO, Scher RK, Li S, et al. Baseline nail disease in patients with moderate to severe psoriasis and response to treatment with infliximab during 1 year. J Am Acad Dermatol. 2008;58(2):224-31.

24. Young MS, Horn EJ, Cather JC. The ACCEPT study: ustekinumab versus etanercept in moderate-to-severe psoriasis patients. Expert Rev Clin Immunol. 2011;7(1):9-13.

25. Rivera R, García-Doval I, Carretero G, Dauden E, SánchezCarazo J, Ferrandiz C, et al. BIOBADADERM: registro español de acontecimientos adversos de terapias biológicas en Dermatología. Primer informe. Actas Dermosifiliogr. 2011; 102(2):132-141. 
26. Leonardi CL, Powers JL, Matheson RT, Goffe BS, Zitnik R, Wang A, et al. Etanercept as monotherapy in patients with psoriasis. N Engl J Med. 2003; 349(21):2014-22.

27. Papp K, Poulin Y, Bissonnette R, Bourcier M, Toth D, Rosoph $L$, et al. Assessment of the long-term safety and effectiveness of etanercept for the treatment of psoriasis in an adult population. J Am Acad Dermatol. 2012;66(2):e33-45.

28. Reich $\mathrm{K}$, et al. The long-term safety of adalimumab: an analisis of all adalimumab exposure in all moderate to severe psoriasis clinical trials. P (3336) en AAD Congress, 2011, Nueva Orleans, EE.UU.

29. Reich K, Nestle FO, Papp K, Ortonne JP, Evans R, Guzzo C, et al. Infliximab induction and maintenance therapy for moderate-to-severe psoriasis: a phase III, multicentre, double-blind trial. Lancet. 2005;366(9494): 1367-74.

30. Reich K, Leonardi C, Griffiths CEM, Szapari PO, Wasi Y, Hsu $\mathrm{MC}$, et al. Update on the cumulative safety experience of ustekinumab: results from the ustekinumab psoriasis clinical development program with up to 4 years of follow-up. Póster FC0703 presentado en el World Congress of Dermatology 2011, Seoul.

31. Tubach F, Salmon D, Ravaud P, Allanore Y, Goupille P, Bréban $\mathrm{M}$, et al. Risk of tuberculosis is higher with anti-tumor necrosis factor monoclonal antibody therapy than with soluble tumor necrosis factor receptor therapy: The three-year prospective French Research Axed on Tolerance of Biotherapies registry. Arthritis Rheum. 2009;60(7):1884-94

32. Mehta NN, Azfar RS, Shin DB, Neimann AL, Troxel AB, Gelfand JM. Patients with severe psoriasis are at increased risk of cardiovascular mortality: cohort study using the General Practice Research Database. Eur Heart J. 2010;31(8): 1000-6.

33. Gelfand JM, Troxel AB, Lewis JD, Kurd SK, Shin DB, Wang X, et al. The risk of mortality in patients with psoriasis: results from a population-based study. Arch Dermatol. 2007;143(12): 1493-9.

34. Wakkee M, Herings RM, Nijsten T. Psoriasis may not be an independent risk factor for acute ischemic heart disease hospitalizations: results of a large population-based Dutch cohort. J Invest Dermatol. 2010;130(4):962-7.

35. Gordon K, Szapary P, Langley RG, Reich K, Lebwohl M, Guzzo C, et al. Effect of ustekinumab on cardiovascular events: results from pooled phase 2 and 3 psoriasis trials. [Poster] (3305) en AAD Congress, 2011, Nueva Orleans, EE.UU.

36. Elliott M, Benson J, Blank M, Brodmerkel C, Baker D, Sharples $\mathrm{KR}$, et al. Ustekinumab: lessons learned from targeting interleukin-12/23p40 in immune-mediated diseases. Ann N Y Acad Sci. 2009;1182:97-110.

37. Farhi D. Ustekinumab for the treatment of psoriasis: review of three multicenter clinical trials. Drugs Today (Barc). 2010; 46(4):259-64.

38. Nathan DM, Angus PW, Gibson PR. Hepatitis B and C virus infections and anti-tumor necrosis factor-alpha therapy: guidelines for clinical approach. J Gastroenterol Hepatol. 2006;21(9):1366-71.

39. Tyring S, Poulin Y, Langley RG, Gordon K, Gottlieb AB, Chiou $C F$, et al. Safety and efficacy profiles of etanercept for up to 144 weeks in patients with moderate to severe plaque psoriasis. Washington: AAD; 2007. p. 2731.

40. Brunasso AM, Puntoni M, Gulia A, Massone C. Safety of antitumour necrosis factor agents in patients with chronic hepatitis C infection: a systematic review. Rheumatology (Oxford). 2011;50(9):1700-11.
41. Herranz P, Sendagorta E, Casado B, Gómez C, Alonso ML, Casado M. Psoriasis en pacientes con infección HIV-VHC. Primer año de tratamiento con ustekinumab. [Póster] en Congreso Nacional de Dermatología 2011 en Santiago de Compostela.

42. Tyring S, Gordon KB, Poulin Y, Langley RG, Gottlieb AB, Bunn $\mathrm{M}$, et al. Long-term safety and efficacy of $50 \mathrm{mg}$ of etanercept twice weekly in patients with psoriasis. Arch Dermatol. 2007;143(6):719-26.

43. Puig L, Dauden E, Carrascosa JM. Comentarios a las directrices europeas y británicas sobre el tratamiento de la psoriasis. Actas dermosifiliogr. 2010;101(4):285-90.

44. Borrego L. Etanercept en el embarazo y lactancia. Actas Dermosifiliogr. 2010;101 Suppl 1:97-101.

45. Ben-Horin S, Yavzori M, Katz L, Picard O, Fudim E, Chowers $\mathrm{Y}$, et al. Adalimumab level in breast milk of a nursing moter (carta). Clin Gastroenterol Hepatol. 2010;8(5):475-6.

46. Van Lümig P, Driessen R, Berends M, Boezeman ], van de Kerkhof $P$, de Jong E. Safety of treatment with biologics for psoriasis in daily practice: 5-year data. J Eur Acad Dermatol Venereol. 2012;26(3):283-91.

47. Papp K, Ho Y, Yeilding N, Szapary PO, Hsu MC, Pouling Y, et al. Malingnancies in ustekinumab- treated moderate to severe psoriasis patients: observations with up to 3 years of followup and comparison to the general United States population. [Póster] (554) en 2010, París.

48. Rodríguez-Valverde V, Caliz R, Alvaro-García JM, Marenco JL, Mulero J, Tornero J, et al. III actualización del consenso de la Sociedad Española de Reumatología sobre terapia biológica en la artritis reumatoide. Reumatol Clin. 2006;2 Supl 2:S52-9.

49. Segal BM, Constantinescu CS, Raychaudhuri A, Kim L, FidelusGort R, Kasper LH. Repeated subcutaneous injections of IL12/23 p40 neutralising antibody, ustekinumab, in patients with relapsing-remitting multiple sclerosis: a phase II, double-blind, placebo-controlled, randomised, dose-ranging study. Lancet Neurol. 2008;7(9):796-804.

50. Mössner R, Reich K. Infliximab in the treatment of psoriasis. Expert Rev Dermatol: 2006;1(4):515-526.

51. Aringer M, Graninger W, Steiner G, Smolen J. Safety and efficacy of tumor necrosis factor alpha blockade in systemic lupus erythematosus: an open-label study. Arthritis Rheum. 2004; 50(10):3161-9.

52. Benson JM, Sachs CW, Treacy G, Zhou H, Pendley CE, Brodmerkel CM, et al. Therapeutic targeting of the IL-12/23 pathways: generation and characterization of ustekinumab. Nat Biotechnol. 2011;29(7):615-624.

53. Lebwohl M, Yeilding N, Szapary P, Wang Y, Li S, Zhu Y, et al. Impact of weight on the efficacy and safety of ustekinumab in patients with moderate to severe psoriasis: rationale for dosing recommendations. J Am Acad Dermatol. 2010;63(4): 571-9.

54. Williams VL, Cohen PR. TNF alpha antagonist-induced lupuslike syndrome: report and review of the literature with implications for treatment with alternative TNF alpha antagonists. Int J Dermatol. 2011;50(5):619-25.

55. Ramos-Casals M, Brito-Zerón P, Muñoz S, Soria N, Galiana D, Bertolaccini L, et al. Autoimmune diseases induced by TNFtargeted therapies: analysis of 233 cases. Medicine (Baltimore) 2007;86(4):242-51

56. Puig L. Obesity and psoriasis: body weight and body mass index influence the response to biological treatment. J Eur Acad Dermatol Venereol. 2011;25(9):1007-11. 